\title{
Zukunft Regionale Versorgung - Forschung, Innovation, Kooperation
}

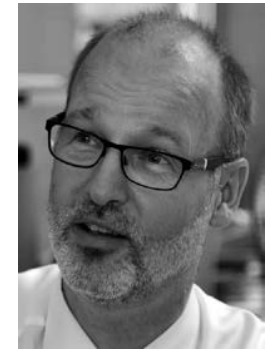

Prof. Wolfgang Hoffmann

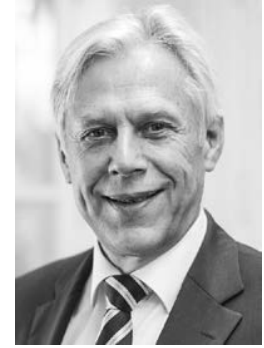

Prof. Dr. Edmund A. M. Neugebauer
Korrespondenzadresse

Prof. Dr. med. Wolfgang Hoffmann, MPH

Institut für Community Medicine

Universitätsmedizin Greifswald

Ellernholzstraße $1 / 2$

17487 Greifswald

wolfgang.hoffmann@uni-greifswald.de

Bibliografie

DOI https://doi.org/10.1055/s-0043-118550

Gesundheitswesen 2017; 79: 814-815

(c) Georg Thieme Verlag KG Stuttgart · New York

ISSN 0941-3790
Liebe Kolleginnen und Kollegen,

sehr geehrte Damen und Herren,

die Versorgungsforschung entwickelt sich derzeit rasch und dynamisch, wir erleben eine Professionalisierung der Versorgungsforscherlnnen und gleichzeitig eine wachsende Wahrnehmung unserer Forschung bei den Akteuren des Gesundheitssystems und in der Gesundheitspolitik. Deutschlandweit wurden große Förderprogramme in der Versorgungsforschung ausgeschrieben. Das BMBF stellt insgesamt 50 Millionen Euro für den „Aktionsplan Versorgungsforschung“ bereit, mit denen Pogramme zum Aufbau von Zentren der gesundheitsökonomischen Forschung, Strukturförderung im Bereich Altersforschung, Kooperationsnetze für die Versorgungsforschung und zuletzt der Aufbau forschungsbezogener Register gefördert werden. Infrastrukturaufbau und wichtige Impulse für die Versorgungsforschung an den Universitätsklliniken werden auch durch die Konsortien der Medizininformatik-Initiative des BMBF geleistet.

An vielen Universitäten und Fachhochschulen wurden neue Lehrstühle und Professuren für Versorgungsforschung eingerichtet, und diese Kolleglnnen empfinden und organisieren sich zunehmend als Fachgruppe. Gleichzeitig wächst erfreulich das Angebot an neuen Studiengängen und umfaßt derzeit BA und Master-Angebote, die deutschlandweit von einer wachsenden Zahl von akademischen Instutionen sowohl als Aufbaustudium als auch grundständig angeboten werden.

Mit einem jährlichen Fördervolumen von 300 Millionen Euro bis 2019 stellt der Innovationsfonds des Gemeinsamen Bundesausschusses einen weiteren Meilenstein in der öffentlichen Finanzierung der Entwicklung neuer Versorgungskonzepte dar. Im Förderzeitraum stehen aus Mitteln der Gesetzlichen Krankenversicherung jährlich 225 Millionen Euro zur Förderung neuer Versorgungsfor- men zur Verfügung, mit weiteren 75 Millionen Euro werden wissenschaftliche Vorhaben aus der Versorgungsforschung gefördert. Eine beeindruckende Zahl von Anträgen wurde geprüft und bewilligt - die Chance für Innovationen ist real. Jetzt sind wir mit dafür verantwortlich, dass aus der beherzten Entscheidung der Politik ein Erfolg für die Versorgung wird!

Das Deutsche Netzwerk Versorgungsforschung wird im SGB V $\S 137 a$, Abs. 7 ausdrücklich als zu beteiligende Institution in Aufträge des IQTIG involviert. Mit seinen Arbeits- und Fachgruppen, seinen Fachgesellschaften, Institutionen und Einzelmitgliedern wird das DNVF regelmäßig zur Stellungnahme zu neuen Gesetzesvorhaben aufgefordert. Die erhöhte wissenschaftliche Wahrnehmung und die Übertragung gestaltender Aufgaben verpflichtet uns auf der anderen Seite, unsere Methoden weiterzuentwickeln, die Qualität und Transparenz unserer Arbeit weiter zu erhöhen, Standards für das eigene Fach zu setzen - und diese dann auch in der Praxis zu leben und in offenen und kooperativen Verfahren weiterzuentwickeln.

In diesem Heft präsentiert die Arbeitsgruppe Qualitäts- und Patientensicherheitsforschung des Deutschen Netzwerkes für Versorgungsforschung (DNVF) e.V. ein mit einer großen Anzahl von Fachgesellschaften konsentiertes neues Methodenmemorandum: DNVF-Memorandum III „Methoden für die Versorgungsforschung“, Teil 3: Methoden der Qualitäts- und Patientensicherheitsforschung, das wesentliche methodische Vorgehensweisen sowie Werkzeuge und Konzepte beschreibt. Eine Kurzfassung ist mit abgedruckt, die Langfassung mit sämtlichen Quellenangaben erscheint gleichzeitig als Open-Access-Publikation online zum kostenlosen Download. Die Qualitäts- und Patientensicherheitsforschung (QPSF) ist ein Kerngebiet der Versorgungsforschung und untersucht den Zusammenhang von Rahmenbedingungen, Strukturen und Prozessen mit Ergebnissen für Personen und Personengruppen in der Gesundheitsversorgung. 
Weiter in diesem Heft findet sich eine Reihe aktueller Themen und Studien zu Mindestmengen und Krankenhaussterblichkeit, zu Notfallthemen wie Inanspruchnahme zentraler Notaufnahmen, zur Allgemeinmedizin in einer universitären Notaufnahme sowie zur Dringlichkeit von hausärztlichen Notfallbesuchen in Altenheimen. Eine systematische Übersichtsarbeit zum Thema Zweitmeinung zeigt, dass es kaum aktuelle Daten zum Effekt von Zweitmeinungsverfahren gibt. Hier ist der G-BA gesetzlich aufgefordert, die Umsetzung dieses Patientenrechtes voranzutreiben.

Dieses Schwerpunktheft erscheint rechtzeitig zum 16. Deutschen Kongress für Versorgungsforschung vom 4.-6. Oktober 2017 in der Berliner Urania. Der Kongress greift die Herausforderungen auf, vor denen unser Gesundheitswesen steht - sektorübergreifende Versorgungsmodelle, professionelle Arbeitsteilung zwischen den medizinischen Berufen, Patientenorientierung auf allen Ebenen der Versorgung, Entwicklung prioritärer Versorgungsziele und -leitlinien, Definition von relevanten Qualitätsindikatoren auf der Basis von Ergebnissen bei den betroffenen Patienten, Implementation praxistauglicher Maße für Effektivität und Effizienz, Digitalisierung und eHealth. Patienten, Akteure im Gesundheitswesen und Gesundheitspolitik erwarten von uns gute und tragfähige Konzepte für ein lernendes, nachhaltiges Gesundheitssystem. Übergreifendes Thema des Kongresses ist „Zukunft Regionale Versorgung
- Forschung, Innovation, Kooperation“: Hier Probleme zu addressieren und Lösungen zu entwickeln, zu implementieren und zu evaluieren, sind originäre Aufgaben der Versorgungsforschung.

Dies gelingt nur im Dialog und Diskurs von Wissenschaft, Praxis und Politik: Seit Jahren hat sich der DKVF als wichtige Plattform für den Austausch von VersorgungsforscherInnen mit VertreterInnen der Selbstverwaltung und aller medizinischen Berufe und gesundheitspolitischen Entscheidern entwickelt und etabliert. In diesem Jahr werden wir an das Erreichte anknüpfen und mit Umstellung auf einen Abstractkongress, neuen Themen und Formaten sowie einer verstärkten internationalen Perspektive die Zukunft der Versorgungsforschung mitgestalten.

Für die VersorgungsforscherInnen gibt es viel - und in Zukunft noch mehr - zu tun, um das Gesundheitswesen von morgen evidenzbasiert, patientenorientiert, qualitätszentriert und nachhaltig zu gestalten. Dabei ist „Das Gesundheitswesen“ seit vielen Jahren ein verlässlicher und professioneller Partner. Wir freuen uns, dass diese Ausgabe die wichtigen Themen Qualität und Patientensicherheit sowie den Innovationsfonds fokussiert und wünschen eine spannende Lektüre.

Ihr Wolfgang Hoffmann und Edmund Neugebauer 